\title{
Tractography-guided surgery of brain tumours: what is the best method to outline the corticospinal tract?
}

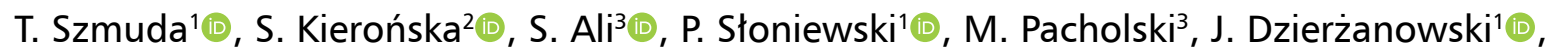
A. Sabisz ${ }^{4}$, E. Szurowska ${ }^{4}$

${ }^{1}$ Neurosurgery Department, Medical University of Gdansk, Poland

${ }^{2}$ Neurology and Neurosurgery Department, University Hospital Collegium Medicum Nicolaus Copernicus University,

Bydgoszcz, Poland

${ }^{3}$ Student's Scientific Circle, Neurosurgery Department, Medical University of Gdansk, Poland

${ }^{4}$ Radiology Department, Medical University of Gdansk, Poland

[Received: 7 January 2020; Accepted: 27 January 2020]

Background: Diffusion tensor imaging (DTI) is the imaging technique used in vivo to visualise white matter pathways. The cortico-spinal tract (CST) belongs to one of the most often delineated tracts preoperatively, although the optimal DTI method has not been established yet. Considering that various regions of interests (ROIs) could be selected, the reproducibility of CST tracking among different centres is low. We aimed to select the most reliable tractography method for outlining the CST for neurosurgeons.

Materials and methods: Our prospective study consisted of 32 patients $(11$ males, 21 females) with a brain tumour of various locations. DTI and T1-weighed image series were acquired prior to the surgery. To draw the CST, the posterior limb of the internal capsule (PLIC) and the cerebral peduncle (CP) were defined as two main ROIs. Together with these main ROIs, another four cortical endpoints were selected: the frontal lobe (FL), the supplementary motor area (SMA), the precentral gyrus (PCG) and the postcentral gyrus (POCG). Based on these ROIs, we composed ten virtual CSTs in DSI Studio. The fractional anisotropy, the mean diffusivity, the tracts' volume, the length and the number were compared between all the CSTs. The degree of the CST infiltration, tumour size, the patients' sex and age were examined.

Results: Significant differences in the number of tracts and their volume were observed when the PLIC or the CP stood as a single ROI comparing with the twoROI method (all $p<0.05)$. The mean CST volume was $40054 U$ (SD \pm 12874$)$ and the number of fibres was 259.3 (SD \pm 87.3 ) when the PLIC was a single ROI. When the CP was a single ROI, almost a half of fibres (147.6; SD \pm 64.0$)$ and half of the CST volume (26664U; SD $\pm 10059 U$ ) was obtained (all $p<0.05$ ). There were no differences between the various CSTs in terms of fractional anisotropy, mean diffusivity, the apparent diffusion coefficient, radial diffusivity and the tract length $(p>0.05)$. The CST was infiltrated by a growing tumour or oedema in 17 of 32 patients; in these cases, the mean and apparent diffusion of the infiltrated CST was significantly higher than in uncompromised CSTs $(p=0.04)$. CST infiltration did not alter the other analysed parameters (all $p>0.05)$. 
Conclusions: A universal method of DTI of the CST was not developed. However, we found that the CP or the PLIC (with or without FL as the second ROI) should be used to outline the CST. (Folia Morphol 2021; 80, 1: 40-46)

Key words: diffusion tensor imaging, diffusion tensor tractography, tractography, corticospinal tract, pyramidal tract, glioma surgery, neurosurgery

\section{INTRODUCTION}

A neurosurgeon's objective is to achieve maximal tumour resection without producing new neurological defects [24, 29]; this includes preserving the cortico-spinal tract (CST). The CST is a white matter bundle that together with corticobulbar tract composes the pyramidal tract and if damaged it may lead to postoperative paresis [6]. Proper preoperative visualisation of the CST and its integration via an intraoperative neuronavigation system could potentially preserve neurological function and simultaneously increase the resection rate $[4,24]$. However, several technical obstacles underlie correct delineation of the CST, including: knowledge of an individual's topographical anatomy, a physician's experience and the degree of destruction of the neuronal pathway through the tumour [14, 21].

Magnetic resonance imaging (MRI) scans are routinely acquired when planning brain tumour surgery. Yet, they provide only general information about the brain's pathology and the surrounding structures. Diffusion tensor imaging (DTI) - which provides a quick and non-invasive method for visualising structural changes of the white matter - can be used for visualisation of the CST $[10,12]$. This technique not only plays an important role in neurosurgical planning but may predict the extent of safe resection [1, 22, 27]. However, physicians currently select various regions of interest (ROI) to draw the CST as the optimal DTI-derived method to estimate the course of the CST has not yet been established [11]. Due to this lack of standardisation the reliability of tracking the CST in different centres remains low [21].

Our aim was to determine the optimal DTI-derived method to reconstruct the CST as well as to verify if a single ubiquitous method exists. To accomplish this, we compared CST tracking as determined by various ROIs.

\section{MATERIALS AND METHODS}

\section{Patients}

Thirty-two patients with a brain tumour invading the CST were prospectively collected (11 males,
21 females; age 27-81 years, mean \pm standard deviation (SD) and median age: $53.4 \pm 17.1 / 53.5)$. These patients were treated at the Neurosurgery Department in Gdańsk, Poland, from 2016 to 2019. The protocol of the study was approved by the local bioethical committee, permission number: NKBBN/65/2019.

\section{Image acquisition}

All patients had a preoperative MRI with a DTI sequence preformed on a 1.5T Siemens Magnetom Aera scanner (Erlangen, Siemens Medical Solutions, Erlangen, Germany) which was equipped with a 20-channel head coil. The standard imaging protocol for brain tumours covered T1, T2-weighed sequences and T1-weighed post-gadolinium which served as a neuronavigational sequence. Diffusion-weighted imaging (DWI) was obtained using: three repetitions of 20 directions, a b-factor of $1000 \mathrm{~s} / \mathrm{m}^{2}$, a slice thickness of $5.0 \mathrm{~mm}$, a $128 \times 128$ matrix, a $240 \times 240 \mathrm{~mm}$ field-of-view, a repetition time of $3500 \mathrm{~ms}$, and an echo time of $83.0 \mathrm{~ms}$. A multivariate linear fitting was used to calculate the orientation for a single voxel. The largest eigenvalue denoted the ultimate fibre direction. A total of 60 diffusion sampling directions were acquired. The in-plane resolution was $1.95313 \mathrm{~mm}$. The slice thickness was $2 \mathrm{~mm}$. The diffusion tensor was calculated and a deterministic fibre tracking algorithm was used [28]. The angular threshold was 90 degrees. The step size was $0.977 \mathrm{~mm}$. Diffusion images were processed and all the analyses were conducted using DSI Studio (dsi-studio.labsolver.org). We calculated the fractional anisotropy (FA), the mean diffusivity (MD) and the apparent diffusion coefficient (ADC). The anisotropy threshold was determined automatically by the software. The fibre trajectories were smoothed by averaging the propagation direction with $30 \%$ of the previous direction. Tracks with a length less than $30 \mathrm{~mm}$ were discarded. A total of 15000 tracts were calculated. When reconstructing the CST, we obtained tract statistics, including: the number of tracks, the mean length, the volume of the CST, the FA, the AD, and the MD values. The ROI 


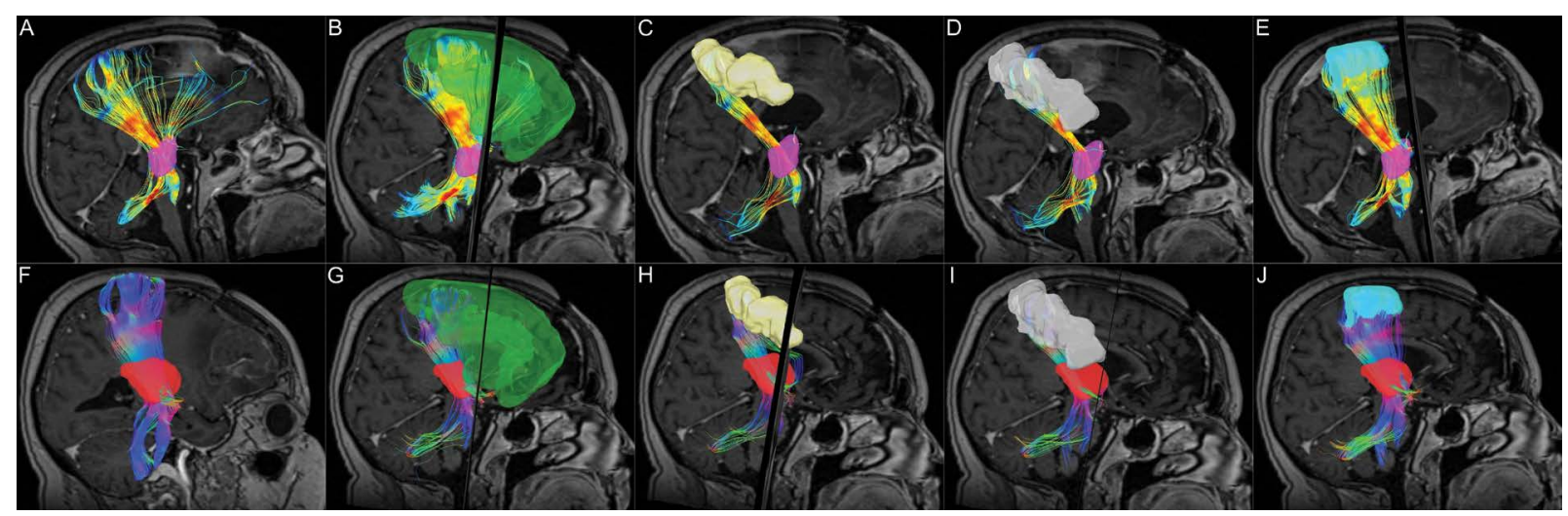

Figure 1. A graphic representation of different ways of drawing the cortico-spinal tract, where its course is determined by various regions of interest; A-E. Cerebral peduncle (CP, violet); F-J. Posterior limb of internal capsule (PLIC, red). A. Only CP; B. CP and frontal lobe (green); C. CP and precentral gyrus (yellow); D. CP and postcentral gyrus (white); E. CP and supplementary motor area (blue). F. Only PLIC; G. PLIC and frontal lobe (green); H. PLIC and precentral gyrus (yellow); I. PLIC and postcentral gyrus (white); J. PLIC and supplementary motor area (blue).

Table 1. A summary of the average values of the number of tracks of ten corticospinal tracts obtained by a combination of various region-of-interests

\begin{tabular}{lcccccc}
\hline ROI & \multicolumn{5}{c}{ Number of tracts } \\
\cline { 2 - 7 } & Number & Mean & Median & Minimum & Maximum & SD \\
\hline CP & 32 & 147.5625 & 144.5000 & 22.0000 & 309.0000 & 64.01257 \\
CP_preCG & 31 & 31.3548 & 24.0000 & 0.0000 & 211.0000 & 40.43888 \\
CP_postCG & 32 & 15.1250 & 12.0000 & 0.0000 & 63.0000 & 15.14819 \\
CP_SMA & 32 & 12.7500 & 10.0000 & 0.0000 & 46.0000 & 13.77000 \\
CP_FL & 32 & 71.1563 & 71.0000 & 3.0000 & 161.0000 & 41.26321 \\
PLIC & 32 & 259.2500 & 248.5000 & 126.0000 & 487.0000 & 87.34581 \\
PLIC_preCG & 32 & 42.3438 & 39.0000 & 1.0000 & 110.0000 & 27.32199 \\
PLIC_postCG & 32 & 24.0938 & 22.0000 & 2.0000 & 88.0000 & 20.76654 \\
PLIC_SMA & 32 & 22.4062 & 18.5000 & 0.0000 & 89.0000 & 21.31614 \\
PLIC_FL & 32 & 115.5938 & 111.0000 & 0.0000 & 287.0000 & 65.03640 \\
\hline
\end{tabular}

$\mathrm{ROI}$ - region of interest, $\mathrm{CP}$ — cerebral peduncle, preCG — precentral gyrus, postCG — postcentral gyrus, SMA — supplementary motor area, FL — frontal lobe, PLIC — posterior limb of internal capsule; SD — standard deviation

was selected according to brain landmarks that the CST passed through and the anatomical automatic atlas provided by DSI-Studio. Hence, we verified again all patients and tested methods which were used for drawing CST by other researchers.

After reviewing recent methods of DTI of the CST, we utilised two basic types of ROIs to outline the CST $[4,11,13,14,19,21,29]$. Two main ROls were designated as "start points": the cerebral peduncle (CP) and the posterior limb of internal capsule (PLIC). The following were established as "end points": the precentral gyrus (PCG), the postcentral gyrus (POCG), the supplementary motor area (SMA) and the frontal lobe (FL). By mixing and matching various combinations of the start point and the end point we obtained ten different CSTs for analysis. An example is illustrated on Figure 1.

\section{RESULTS}

The CST was reconstructed by 10 different algorithms for each of the 32 patients in the study, resulting in a total of 320 outlines of the CST. The fibres were visualized with DSI-studio to show the anatomical validity of the reconstruction results and we overlay the CST fibres bundles with the FA image. Although the CST was outlined in every patient, a few selected variables could not be calculated due to unknown internal software error(s). The average number of tracts that were obtained with the ten algorithms are summarised in Table 1. 


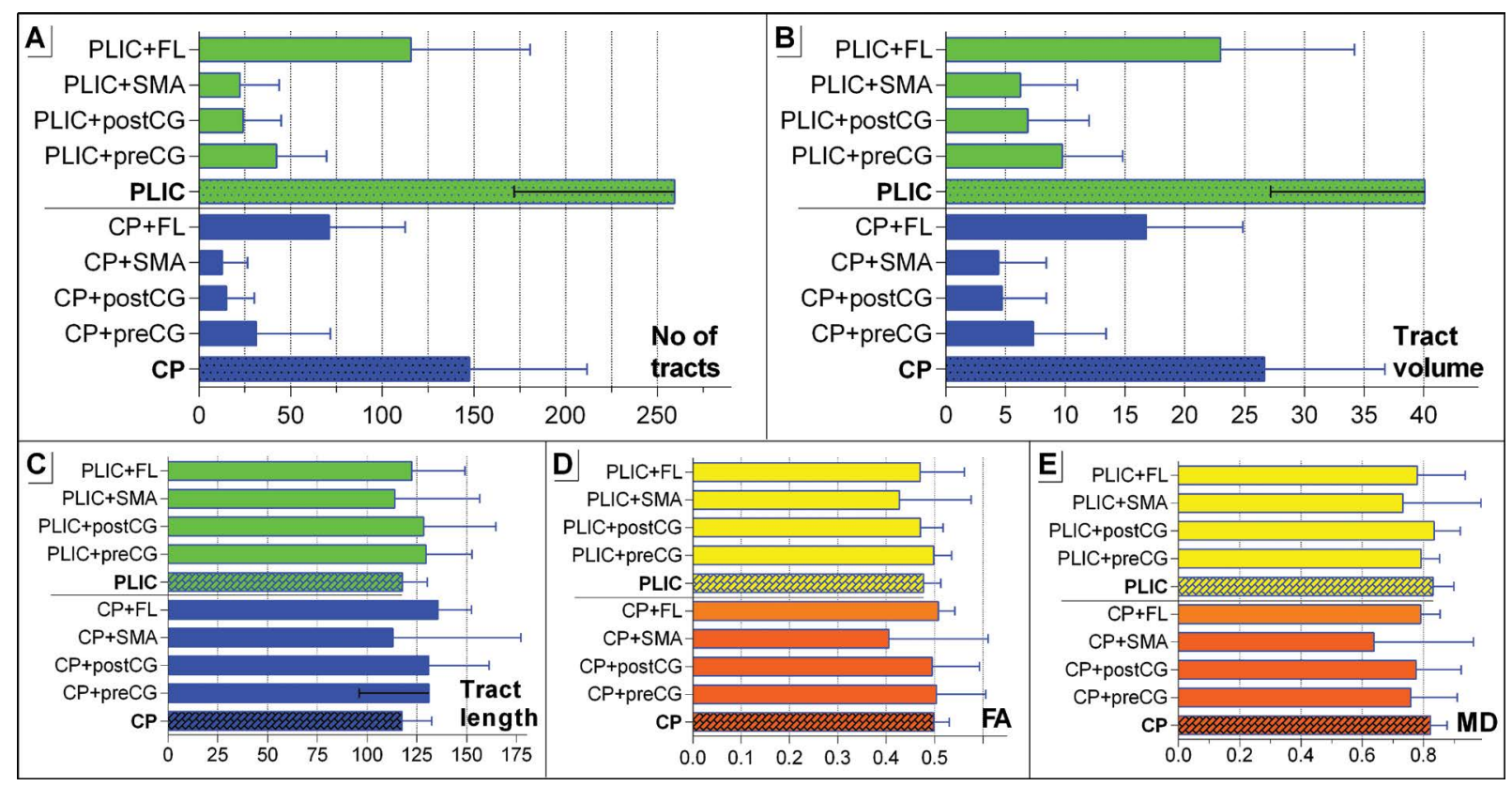

Figure 2. The illustration summarises the findings when using 10 different methods of outlining the corticospinal tract, including the mean; A. Number of tracts; B. Tract volume; C. Tract length; D. Fractional anisotropy (FA); E. The mean diffusivity (MD); PLIC — posterior limb of internal capsule, $\mathrm{CP}$ - cerebral peduncle, preCG — precentral gyrus, postCG — postcentral gyrus, SMA — supplementary motor area, $\mathrm{FL}$ - frontal lobe.

A significantly higher volume (40054U; SD \pm $\pm 12874 \mathrm{U})$ and number of CST tracts (259.3; SD \pm \pm 87.3 ) were obtained when a single $\mathrm{ROI}$ was set at the PLIC comparing to all other ROIs (all $p<0.05$ ). In contrast, almost $50 \%$ less fibres (147.6; SD \pm 64.0$)$ and volume (26664U; SD $\pm 10059 \mathrm{U})$ was achieved when the $\mathrm{CP}$ was set as the ROI (both $p<0.05$ ) rather than the PLIC. Altogether, the PLIC and CP as starting points comprised a 6.1 -fold and 4.7 -fold greater number of fibres, respectively than the most common anatomical course of the CST (first ROI set at CP or PLIC, and the cortical ROI set at the precentral gyrus [preCG]; $p<0.01$ ).

Setting the $\mathrm{FL}$ as the endpoint $\mathrm{RO}$ yielded the highest number of tracts (PLIC and FL: 115.6; SD \pm 65.0 ) and the highest tract volume (CP and FL: 71.1; SD \pm 41.3 ).

There were no significant differences between the various CSTs in terms of the mean diffusion parameters (FA, MD, apparent diffusion and radial diffusivity) and mean tract length. However, all these values were insignificant when the SMA was set as a ROI (Fig. 2).

Seventeen of the 32 patients (53.1\%) with preoperative hemiparesis had a brain tumour or oedema which infiltrated the CST. Even though the degree of infiltration on the CST was not evaluated, the FA, the number of tracts and the tract volumes did not differ between the CSTs drawn in ten configurations of the ROIs (all $p>0.05$ ). However, in patients with CST infiltration through the $C P$, the MD and the apparent diffusion $(A D)$ was significantly higher than in patients without tract infiltration $(p=0.04)$. This may result from the tumour infiltrating not only the CST but other fibres which are drawn only when the $C P$ is selected as a single ROI. The higher AD was also observed when the postcentral gyrus was added as an additional ROI to the PLIC $(p=0.03)$. This patient group was mostly diagnosed high grade glioblastoma or oligodendroglioma located by the left fronto-parietal lobe, which may explain the finding.

As seen on part $D$ and $E$ on Figure 2, we compared the FA and MD values of the CST between the two brain hemispheres and the patient's sex, yet neither hemisphere yielded significantly different values (all $p>0.05$ ). However, patients older than 55 years had a significantly higher MD when the CST was traced from a start point $\mathrm{ROI}(p=0.031)$, an endpoint ROI 2 (0.036) and a start point ROI with an end point in the FL $(p=0.013)$. All the statistics comparing the 10 methods of tracking the CST are presented on Supplementary Table 1 (see journal website) (Fig. 3).

\section{DISCUSSION}

We found that while there is no universal way of determining the CST, the CP or the PLIC should be 


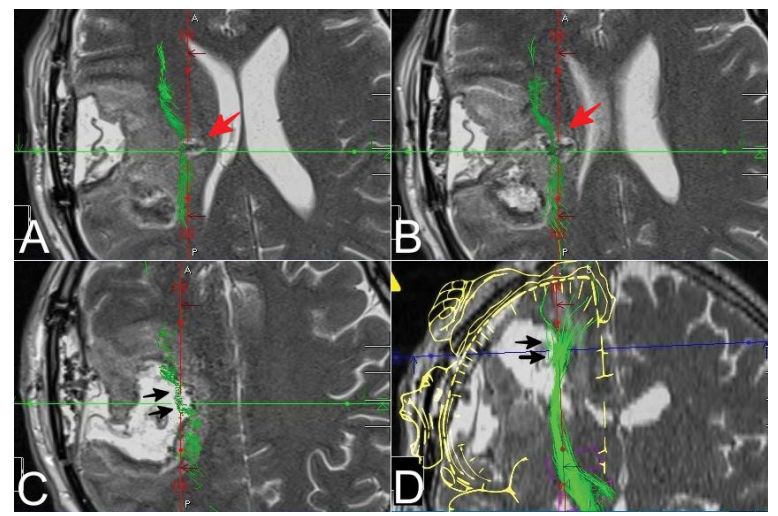

Figure 3. A clinical case presentation where an early postoperative magnetic resonance image was fused with a preoperative diffusion tensor imaging (DTI) of the cortico-spinal tract (CST). The subtotal resection of the secondary glioblastoma of the left fronto-parietal region was considered with the preservation of the fibres of the corticospinal tract to preserve foot function. Therefore, only the posterior limb of the internal capsule was selected as a single region of interest to increase DTI sensitivity, regardless of the decreased parallel specificity as many of fibres did not belong to the CST; A-C. Axial planes; D. Coronal plane, motor homunculus as the injected picture. The red arrows indicate the tumour remnant left deliberately in the CST. The black arrows show the compromised CST fibres responsible for hand movement.

exclusively used as the starting point $\mathrm{ROI}$ - regardless of whether the FL is used as the second ROI. This information is invaluable to neurosurgeons as it empowers them to obtain the most optimal view of the CST using DTI. Allowing a surgeon to better understand the spatial orientation between the tumour and the CST fibres may help preserve the CST and therefore minimize the risk of postoperative paresis; since the CST directs the movement of the limbs and the trunk [17]. To the best of our knowledge our findings are novel as our study is the first to compare the various DTI techniques regarding the CST.

It is an established issue that a long fibre bundle may not be recognised as a real fibre. Chenot proved that by using standard ROIs (i.e. PLIC and $\mathrm{CP}$ ) we obtain a CST far beyond the primary motor cortex [6]. They showed that the CST streamlines from the premotor and parietal cortex. This finding is in contradiction with the well-known course of the pyramidal tract as described by Dejerine in 1901 [7]. To date, the exact origin of CST and the function of its non-primary motor cortex originating fibres remain unclear and unproven [2].

For neurosurgeons, the fibres originating at the precentral gyrus are the most crucial while planning any surgical access near the pyramidal tracts $[10,12$, $23,25]$. A study suggests that the remainder of the
CST fibres come from the FL, mainly from the superior frontal gyrus [6]. Our results confirm this since we added the entire frontal lobe as a second ROI to the PLIC or the CP and reduced the number of fibres and the tract volume approximately 2 -fold. This suggests that choosing the CP or PLIC as a single ROI does seem to be the most optimal solution when conservative access to the brain pathology is planned to minimise damage to the CST. However, limiting the extent of the ROI-based DTI to the "most anatomical" course of the CST (primary motor cortex as a second ROI to $\mathrm{PLIC/CP}$ ), decreases the number of potentially valid fibres 6 -fold as there is high variability of the CST volume among the population $[6,14]$. Thus, there is no universal tractographic approach to delineate the CST due to interference of other crossing fibre tracts (although it may be overcome when the CP or the PLIC stand as a single ROI) [19]. Some authors proposed using the CP and the PLIC as two ROI where all the fibres would run through $[13,29]$. At our institution we abandoned this technique and now use a custom DTI technique for all our patients because we believe that estimating the CST around the tumours at the eloquent cortex should utilise a variety of ROIs rather than a standardised set due to several of the reasons indicated before. Of note, DTI operators almost always refer to the $\mathrm{CP}$ as a reference for tracking the CST; however, this term is slightly ambiguous. The cerebral crus is a more accurate term anatomically as it refers solely to the anterior part of the $\mathrm{CP}$ (which is the correct location for CST tracking).

Setting the ROI at the PLIC was suggested as the approach most resembling the anatomical course of the CST [5]. In our study, the largest volume of the CST was achieved when a single ROI was selected, without indicating any termination points in the brain cortex. In our analysis the ROI point is relatively easy to use and is supported by most DTI data analysis software. The correct ROI may be determined on structural T1-weighed images due to their precisely defined anatomical structures. However, diffusion images that are directionally orientated (DEC sequence) are also useful to define the ROIs especially when white matter structures are being investigated. Thus, special care must be taken when choosing the appropriate ROI [26]. Seeding the termination ROI in a functionally active cortex may be regarded as the most precise method, although, this presurgical task-based functional MRI cannot provide reliable information about the CST in tumours located in the eloquent cortex [15]. 
In tumours located in or infiltrating the motor cortex the volume of interest (volumetric ROI) may not stand as a single cortical ROI for tracking the CST due to low accuracy [15].

Today, computer software offers automatic (anatomical) atlases to a given brain. This atlas-based method or its combination with DTI has proven to be a similar predictor of clinical outcome when compared with traditional DTI of the CST [21]. Moreover, automatic CST recognition may grant an advantage when planning brain tumour surgery. Recently, O'Donnell et al. [16] proposed the automatic patient-specific method for identifying the CST and the arcuate fasciculus and the DTI predictions corresponded with functional MRIs in $94 \%$ of patients. Additionally, the author indicated that the true anatomical termination of the main human tracts remains under debate. Thus, the combination of various techniques, including functional MRI, DTI and computational techniques, could bring scientists closer to discovering the correct CST delineation. On the contrary, the supporters of awake craniotomy for low grade gliomas urge that neuromonitoring and intraoperative stimulation maximize the tumour resection rate while still preserving motor function [8]. Yet, several patients may not be qualified for an awake procedure due to other medical and psychological reasons. We stress that in the absence of functional MRIs, an intraoperative neuromonitoring or motor mapping pathways should be considered to support DTI.

In patients with a high grade glioblastoma, where total resection remains critical, a minor neurological deficit is considered acceptable [24]. In these patients, the CP, the PLIC and the FL were deliberately selected as ROls as they were the most important CST motor fibres that should be preserved during surgery. With this example, we assert that estimating the CST should be patient-specific as these changes in performing DTI are advantageous. Additionally, literature suggests that the CST's trajectory can change in certain brain pathologies such as a stroke, a brain tumour or a subarachnoid haemorrhage [18]. This may lead to significant alteration in the CST's original route where intraoperative direct stimulation cannot be substituted by other imaging techniques. Bello et al. reported that various types of brain tumours cause modification of white matter fibre trajectory. Based on the DTI analysis, a half of high grade gliomas caused dislocation and some tumours infiltrated or interrupted the course of tracts [3]. On the contrary, low grade gliomas only infiltrated or interrupted tracts since only a quarter of patients had dislocated fibres. When DTI was compared with intraoperative subcortical mapping, the results illustrated that the CST's course was determined on the basis of DTI depended tumour location and volume [3].

The appropriate choice between these two ROls lies upon the discretion of the DTI operator. Based on our analysis, seeding the CST from the PLIC results in a significantly greater number of tracks and a larger volume of the CST than seeding from the CP. We postulate that since the CP is smaller than the PLIC, it is less packed in white matter and therefore yields different results [20]. The path of the white matter fibres depends on the degree of precision of the determined ROls. While plotting points may be determined manually or automatically, in our paper, the CST was determined with an automatic anatomical atlas using two- and three-dimensional planes.

Analysing the CST poses several challenges such as the disturbed anisotropy of water molecules and the reorganisation of nerve fibres. Moreover, tissue swelling around the tumour may disturb the proportions of perpendicular and parallel diffusion, which decreases the FA; this is due to the inversely proportional ratio of average diffusivity and cellularity of the pathology [21]. In addition, the measurement of anisotropy may become a factor differentiating tumours of high and low level of malignancy [21].

In future studies, different tractography methods can be connected with DTI multi-tensor acquisition. Identification of the CST should be carried out based on blood-oxygen-level-dependent and diffusion functional MRIs [9]. These methods may help a physician achieve a better regional and spatial understanding of an area.

\section{CONCLUSIONS}

Our study identified the CST based on various anatomical approaches. We found that while there is no universal method of determining the CST, the CP or the PLIC should be used as the starting point ROIs.

\section{REFERENCES}

1. Anthofer JM, Steib K, Fellner C, et al. DTI-based deterministic fibre tracking of the medial forebrain bundle. Acta Neurochir (Wien). 2015; 157(3): 469-477, doi: 10.1007/ s00701-014-2335-y, indexed in Pubmed: 25585836.

2. Archer DB, Vaillancourt DE, Coombes SA. A template and probabilistic atlas of the human sensorimotor tracts using diffusion MRI. Cereb Cortex. 2018; 28(5): 1685-1699, doi: 10.1093/cercor/bhx066, indexed in Pubmed: 28334314.

3. Bello L, Gambini A, Castellano A, et al. Motor and language DTI fiber tracking combined with intraoperative 
subcortical mapping for surgical removal of gliomas. Neuroimage. 2008; 39(1): 369-382, doi: 10.1016/j.neuroimage.2007.08.031, indexed in Pubmed: 17911032.

4. Bonney PA, Conner AK, Boettcher LB, et al. A simplified method of accurate postprocessing of diffusion tensor imaging for use in brain tumor resection. Oper Neurosurg (Hagerstown). 2017; 13(1): 47-59, doi: 10.1227/ NEU.0000000000001181, indexed in Pubmed: 28931252.

5. Bürgel $U$, Amunts $K$, Hoemke $L$, et al. White matter fiber tracts of the human brain: three-dimensional mapping at microscopic resolution, topography and intersubject variability. Neuroimage. 2006; 29(4): 1092-1105, doi: 10.1016/j. neuroimage.2005.08.040, indexed in Pubmed: 16236527.

6. Chenot Q, Tzourio-Mazoyer N, Rheault F, et al. A population-based atlas of the human pyramidal tract in 410 healthy participants. Brain Struct Funct. 2019; 224(2): 599-612, doi: 10.1007/s00429-018-1798-7, indexed in Pubmed: 30460551.

7. Dejerine J, Dejerine-Klumpke A. Anatomie des centres nerveux. Tome 2. Rueff et Cie, Paris 1901.

8. Duffau H, Taillandier L. New concepts in the management of diffuse low-grade glioma: Proposal of a multistage and individualized therapeutic approach. Neuro Oncol. 2015; 17(3): 332-342, doi: 10.1093/neuonc/nou153, indexed in Pubmed: 25087230.

9. Holodny Al, Ollenschleger MD, Liu WC, et al. Identification of the corticospinal tracts achieved using blood-oxygen-level-dependent and diffusion functional MR imaging in patients with brain tumors. Am J Neuroradiol. 2001; 22(1): 83-88, indexed in Pubmed: 11158892.

10. Kieronska $S$, Stoniewski P. The usefulness and limitations of diffusion tensor imaging - a review study. Eur J Transl Clin Med. 2020; 2(2): 43-51, doi: 10.31373/ejtcm/112437.

11. Kim B, Fisher BE, Schweighofer $N$, et al. A comparison of seven different DTI-derived estimates of corticospinal tract structural characteristics in chronic stroke survivors. J Neurosci Methods. 2018; 304: 66-75, doi: 10.1016/j. jneumeth.2018.04.010, indexed in Pubmed: 29684462.

12. Krakowiak M, Słoniewski P, Dzierżanowski J, et al. Future of the nerve fibres imaging: tractography application and development directions. Folia Morphol. 2015; 74(3): 290-294, doi: 10.5603/FM.2015.0044, indexed in Pubmed: 26339808.

13. Kuczynski AM, Dukelow SP, Hodge JA, et al. Corticospinal tract diffusion properties and robotic visually guided reaching in children with hemiparetic cerebral palsy. Hum Brain Mapp. 2018; 39(3): 1130-1144, doi: 10.1002/ hbm.23904, indexed in Pubmed: 29193460.

14. Leote J, Nunes RG, Cerqueira L, et al. Reconstruction of white matter fibre tracts using diffusion kurtosis tensor imaging at 1.5T: Pre-surgical planning in patients with gliomas. Eur J Radiol Open. 2018; 5: 20-23, doi: 10.1016/j. ejro.2018.01.002, indexed in Pubmed: 29719853.

15. Niu C, Liu X, Yang $Y$, et al. Assessing region of interest schemes for the corticospinal tract in patients with brain tumors. Medicine (Baltimore). 2016; 95(12): e3189, doi: 10.1097/ MD.0000000000003189, indexed in Pubmed: 27015212.

16. O'Donnell $\sqcup$, Suter $Y$, Rigolo $L$, et al. Automated white matter fiber tract identification in patients with brain tum- ors. Neuroimage Clin. 2017; 13: 138-153, doi: 10.1016/j. nicl.2016.11.023, indexed in Pubmed: 27981029.

17. Ottenhausen $M$, Krieg SM, Meyer $B$, et al. Functional preoperative and intraoperative mapping and monitoring: increasing safety and efficacy in glioma surgery. Neurosurg Focus. 2015; 38(1): E3, doi: 10.3171/2014.10. FOCUS14611, indexed in Pubmed: 25552283.

18. Park CH, Kou N, Boudrias MH, et al. Assessing a standardised approach to measuring corticospinal integrity after stroke with DTI. Neuroimage Clin. 2013; 2: 521-533, doi: 10.1016/j.nicl.2013.04.002, indexed in Pubmed: 24179804.

19. Pujol S, Wells W, Pierpaoli $C$, et al. The DTI challenge: toward standardized evaluation of diffusion tensor imaging tractography for neurosurgery. J Neuroimaging. 2015; 25(6): 875-882, doi: 10.1111/jon.12283, indexed in Pubmed: 26259925.

20. Radmanesh A, Zamani AA, Whalen $S$, et al. Comparison of seeding methods for visualization of the corticospinal tracts using single tensor tractography. Clin Neurol Neurosurg. 2015; 129: 44-49, doi: 10.1016/j.clineuro.2014.11.021, indexed in Pubmed: 25532134.

21. Ressel V, van Hedel HJA, Scheer I, et al. Comparison of DTI analysis methods for clinical research: influence of pre-processing and tract selection methods. Eur Radiol Exp. 2018; 2(1): 33, doi: 10.1186/s41747-018-0066-1, indexed in Pubmed: 30426317.

22. Soni N, Mehrotra A, Behari S, et al. Diffusion-tensor imaging and tractography application in pre-operative planning of intra-axial brain lesions. Cureus. 2017; 9(10): e1739, doi: 10.7759/cureus.1739, indexed in Pubmed: 29209586.

23. Szmuda T, Ali S. Commentary on: The usefulness and limitations of diffusion tensor imaging - a review study. Eur J Transl Clin Med. 2020; 2(2): 85-86, doi: 10.31373/ejtcm/114000.

24. Szmuda T, Słoniewski P, Olijewski W, et al. Colour contrasting between tissues predicts the resection in 5-aminolevulinic acid-guided surgery of malignant gliomas. J Neurooncol. 2015; 122(3): 575-584, doi: 10.1007/ s11060-015-1750-0, indexed in Pubmed: 25702194.

25. Szmuda T, Słoniewski P, Szmuda M, et al. Quantification of white matter fibre pathways disruption in frontal transcortical approach to the lateral ventricle or the interventricular foramen in diffusion tensor tractography. Folia Morphol. 2014; 73(2): 129-138, doi: 10.5603/FM.2013.0063, indexed in Pubmed: 24902089.

26. Van Hecke W, Emsell L, Sunaert S. Diffusion Tensor Imaging: A Practical Handbook. Diffus Tensor Imaging A Pract Handb. 2016: 1-440, doi: 10.1007/978-1-4939-3118-7.

27. Witwer BP, Moftakhar R, Hasan KM, et al. Diffusion-tensor imaging of white matter tracts in patients with cerebral neoplasm. J Neurosurg. 2002; 97(3): 568-575, doi: 10.3171/ jns.2002.97.3.0568, indexed in Pubmed: 12296640.

28. Yeh FC, Wedeen VJ, Tseng WYI. Generalized q-sampling imaging. IEEE Trans Med Imaging. 2010; 29(9): 1626-1635, doi: 10.1109/TMI.2010.2045126, indexed in Pubmed: 20304721.

29. Yu Qi, Lin K, Liu Y, et al. Clinical uses of diffusion tensor imaging fiber tracking merged neuronavigation with lesions adjacent to corticospinal tract : a retrospective cohort study. J Korean Neurosurg Soc. 2020; 63(2): 248-260, doi: 10.3340/jkns.2019.0046, indexed in Pubmed: 31295976. 